\title{
Antioxidants L-carnitine and D-methionine modulate neuronal activity through GABAergic inhibition
}

\author{
Calvin Wu • Kamakshi V. Gopal • Ernest J. Moore • \\ Guenter W. Gross
}

Received: 10 October 2013/Accepted: 30 January 2014

(C) Springer-Verlag Wien 2014

\begin{abstract}
Antioxidants are well known for their neuroprotective properties against reactive oxygen species in cortical neurons and auditory cells. We recently identified L-carnitine and D-methionine to be among agents that provide such protection. Here, we investigated their neuronal modulatory actions. We used cultured neuronal networks grown on microelectrode arrays to assess the effects of L-carnitine and D-methionine on network function. Spike production and burst properties of neuronal networks were used as parameters to monitor pharmacological responses. L-Carnitine and D-methionine reduced spike activity with $100 \%$ efficacy with $\mathrm{EC}_{50}$ values of $0.22( \pm 0.01) \mathrm{mM}$ and $1.06( \pm 0.05) \mathrm{mM}$, respectively. In the presence of $1.0-40 \mu \mathrm{M}$ of the $\mathrm{GABA}_{\mathrm{A}}$ antagonist bicuculline, the sigmoidal concentration-response curves of both compounds exhibited stepwise shifts, without a change in efficacy. Under a maximal bicuculline concentration of $40 \mu \mathrm{M}$, the $\mathrm{EC}_{50}$ increased to $3.57( \pm 0.26) \mathrm{mM}$ for L-carnitine and to $10.52( \pm 0.97) \mathrm{mM}$ for D-methionine, more than a tenfold increase. The agonist-antagonist interactions with bicuculline were estimated by Lineweaver-Burk plot analyses to be competitive, corroborated by the computed dissociation constants of bicuculline. For both compounds, the
\end{abstract}

C. $\mathrm{Wu} \cdot \mathrm{G}$. W. Gross

Department of Biological Sciences, University of North Texas, Denton, TX 76203, USA

C. Wu $(\bowtie) \cdot$ K. V. Gopal · E. J. Moore

Department of Speech and Hearing Sciences, University of

North Texas, 1155 Union Circle \#305010, Denton,

TX 76203-5017, USA

e-mail: calvinwu@my.unt.edu

C. Wu · K. V. Gopal · E. J. Moore · G. W. Gross Center for Network Neuroscience, University of North Texas, Denton, TX 76203, USA effects on the network burst pattern, activity reversibility, and bicuculline antagonism resembled that elicited by the $\mathrm{GABA}_{\mathrm{A}}$ agonist muscimol. We showed that the antioxidants L-carnitine and D-methionine modulate cortical electrical spike activity primarily through $\mathrm{GABA}_{\mathrm{A}}$ receptor activation. Our findings suggest the involvement of GABAergic mechanisms that perhaps contribute to the protective actions of these compounds.

Keywords $\mathrm{GABA}_{\mathrm{A}}$ receptor Bicuculline . Agonist-antagonist interaction - Microelectrode array . Neuroprotection

\section{Introduction}

Oxidative stress due to increased reactive oxygen species (ROS) production is considered one of the major causes of neuronal degeneration (Sesti et al. 2010; Napolitano et al. 2011), and may participate in many PNS or CNS disorders such as hearing loss, Alzheimer disease, Parkinson disease, and epilepsy. For neurons under ROS stress, a variety of essential cellular components undergo peroxidation that leads to metabolic imbalance, impaired mitochondrial function, depletion of the antioxidant system, and disruption of membrane ion conduction (Waldbaum and Patel 2010; Palacios et al. 2011; Nisticò et al. 2011). In the cochlea, increased ROS production is coupled to ototoxicinduced hearing loss (Rybak et al. 2007; Poirrier et al. 2010), with damaging effects extending to the cortical areas of the central auditory pathways, and causing aberrant conditions such as tinnitus (Rybak 2005). Thus, strategies to combat the effects of ROS stress are needed for both prevention and treatment of inflammation, neurodegeneration, and concomitant conditions. 
In recent years, several antioxidants have shown the ability to protect neurons from ROS toxicity and degeneration. L-Carnitine, among a wide array of target neuroprotective agents, is of particular interest in neurons due to its role in amino acid synthesis, ATP metabolism, mitochondrial fatty acid transport, as well as its antioxidant effects (Gülçin 2006; Jones et al. 2010; Nalecz et al. 2004; Silva-Adaya et al. 2008). Studies of murine cortical neurons have shown that L-carnitine effectively blocked neuronal apoptosis induced by increased ROS after anesthesia exposure (Zou et al. 2008), attenuated the effect of methamphetamine-induced neurotoxicity, diminished the effects of other mitochondrial toxins (Virmani et al. 2003), and reduced neuronal loss due to oxidative stress in animals with Huntington's disease (Vamos et al. 2010). Our earlier study also reported the effect of L-carnitine in neurons exposed to the convulsant agent pentylenetetrazol and demonstrated that L-carnitine can be considered a potential candidate to attenuate over-excitation in cortical networks (Wu et al. 2011).

The common result of oxidative stress damage to the inner ear is hearing loss, the causes being primarily noise exposure, aging, genetic predisposition, or ototoxicity from prescribed medications (Henderson et al. 2006; Rybak et al. 2007). In the cochlea, where the injuryinduced ROS level is often cumulative due to the closed system of the inner ear physiology, prevention and protection by antioxidants have been a major focus of past research. Several antioxidants such as sodium thiosulfate, D-methionine, and $\mathrm{N}$-acetylcysteine have been reported to reduce oxidative stress in cochlear tissues and ameliorate hearing loss induced by noise or chemical insults (Blakley et al. 2002; Lorito et al. 2011). Our previous study focused on the neuroprotective properties of D-methionine, and showed that this compound was protective against cisplatin-induced toxicity in cortical networks (Gopal et al. 2012).

While continuing to search for effective neuroprotective agents, it is important to determine the underlying mechanism of action of these target therapeutic agents. Our studies previously alluded to the inhibitory effects of L-carnitine and D-methionine on neuronal activity, as both compounds attenuated over-excitation (Wu et al. 2011; Gopal et al. 2012). We suggest that inhibitory neurotransmission driven by the GABAergic system may be a direct cause of activity attenuation. Using rapid electrophysiological functional assays, we report a novel characteristic of L-carnitine as well as D-methionine, and present data suggesting inhibition of neuronal activity through $\mathrm{GABA}_{\mathrm{A}}$ receptor activation. To our knowledge, this is the first study showing that these key neuroprotective antioxidants, L-carnitine and D-methionine exhibit modulatory responses that strongly implicate the GABAergic system.

\section{Methods}

Neuronal cell culture on microelectrode arrays

All procedures involving animals in this study were approved by and conducted in accordance with the guidelines of the University of North Texas Institutional Animal Care and use Committee. In-house fabrication and preparation of microelectrode arrays have been described in earlier publications (see Gross et al. 1985). A single type of MEA plate, with $1 \times 1 \mathrm{~mm}^{2}$ recording area of an $8 \times 8$ electrode matrix, was used with equidistant electrode spacing of $150 \mu \mathrm{m}$. Standard cell culture techniques have also been described previously for cortical tissues (Gopal and Gross 1996; Keefer et al. 2001). Cortical tissues were dissociated from mouse embryos (Balb-C/ICR, E17), minced, and digested with papain, followed by trituration in Dulbecco's Modified Minimal Essential Medium (DMEM) with $4 \%$ fetal bovine serum and $4 \%$ horse serum. The cell suspension was seeded at $70-80 \mathrm{~K}$ cells/ $100 \mu \mathrm{l}$ with all cell types present in the parent tissue at the time of isolation. Cells were plated onto MEAs - surface coated with poly-D-lysine and laminin-in volumes of $0.1 \mathrm{ml}$ to a $3.0 \mathrm{~mm}$ diameter adhesion island. Cultures were transitioned to $6 \%$ horse serum medium after 5-7 days and maintained biweekly by half medium changes. All media were supplemented with $2 \%$ B-27 (Gibco, Grand Island, NY). The incubator environment was maintained at $10 \% \mathrm{CO}_{2}$ and $90 \%$ air, at $37{ }^{\circ} \mathrm{C}$. The medium was monitored and kept at 300-320 mOsm $/ \mathrm{kg}$. In this study, the average culture age was $31( \pm 1.0)$ days in vitro (range 18-42 days in vitro). No attempt was made to control glial growth with antimitotics.

\section{Electrophysiological recordings}

The recording instrumentation has been described previously (Keefer et al. 2001; Gopal et al. 2007). Microelectrode arrays were assembled into sterile recording chambers consisting of stainless steel chamber blocks mounted on heated base plates (Gross and Schwalm 1994) on an inverted microscope stage. The temperature was maintained at $37 \pm 0.2{ }^{\circ} \mathrm{C}$ by a custom thermocouplecontrolled power supply providing DC current to power resistors on the base plate. After chamber assembly and recording channel assignment, the original medium was replaced by fresh DMEM stock medium without serum. The $\mathrm{pH}$ was maintained at 7.4 with a continuous $10 \mathrm{ml} / \mathrm{min}$ stream of filtered $10 \% \mathrm{CO}_{2}$ in air, confined by a chamber cap that allowed microscope observation during recordings (Gross and Schwalm 1994). A syringe infusion pump (Harvard Apparatus Pump 11) compensated for evaporation due to the dry airflow with sterile water injection of 
approximately $60 \mu \mathrm{l} / \mathrm{h}$ (Rijal and Gross 2008). Drug stock solutions were prepared in water at concentrations that minimized osmolarity changes in the $2.0 \mathrm{ml}$ constant volume experimental bath (usually $<1.0 \%$ of total volume). D-Methionine and L-carnitine were obtained from SigmaAldrich (St. Louis, MO) in powder form. Neuronal activity was recorded with a 64-channel amplifier system (Plexon Inc., Dallas, TX) and digitized simultaneously at $40 \mathrm{kHz}$. Total system gain was set to $1.1 \times 10^{4}$. Spike identification and separation were accomplished with a real-time template-matching algorithm (Plexon Inc., Dallas, TX) to provide single-unit spike rate data. Electrodes/channels were assigned to 64 digital signal processors (DSP). Depending on wave shape and signal amplitude, each DSP could discriminate up to four different action potential waveforms (units) in real time and assign them to separate output channels.

\section{Data analyses}

All electrophysiological activity was displayed over the course of an experiment using total spike rate from all discriminated active units within the culture in 1-min bins. The term "unit" refers to any cellular compartment that generated action potentials of a size that allowed discrimination via template matching and detection via threshold crossing at $\sim 2 \times$ RMS noise. All real-time computer displays plotted also the active channels per minute based on the number of threshold crossings exceeding 10/min for a specific template. For quantification purposes, all data were normalized by calculating percent activity changes from the reference activity established by each respective network under serum free medium. This approach required establishment of drug/receptor equilibrium conditions that are reflected in stable temporal network activity. Transition activity phases after compound additions were ignored and only the horizontal "minute mean" activity plateaus were used for analyses. Compound-induced activity was therefore normalized and expressed as percent decrease from the internal reference. This approach provided reproducible concentration-response curves obtained from different networks, and also allowed for calculation of dissociation constants (Rijal and Gross 2008). Methods for burst identification have been described previously (Morefield et al. 2000). Briefly, bursts were derived from spike integration with a time constant of $100 \mathrm{~ms}$ for each discriminated unit. The integrated burst profiles were quantified using two thresholds: the first threshold marked the starting point of the burst, while the second threshold determined if the burst profile size were sufficient to be included in the analysis. Threshold levels were determined off-line by comparing integrated burst identification with visual inspection of the raster plots that show the actual burst activity for the same time period. Two parameters of burst were quantified in this study: burst period (interval from the beginning of a burst to the beginning of the next burst) and burst duration (time span of spike occurrence within a burst). Drug-induced changes in burst duration and period were normalized and compared across different networks. All average values were expressed as mean and standard error. The number of observation $(n)$ listed in each result indicated the number of individual networks used-a total of $n=69$ networks were used in this study. Statistical analyses, curve fitting, and linear and nonlinear regressions were accomplished with Prism (Graphpad, La Jolla, CA). Significance was established by analysis of variance (ANOVA) with Tukey's post hoc tests at a significance level of 0.05 .

\section{Results}

Neuronal network responses to L-carnitine and D-methionine

The real-time data of a typical frontal cortex network responding to increasing concentrations of L-carnitine and D-methionine are displayed in Fig. 1a, b, as total network spike production/min. After transition from a serum-containing to a serum-free medium, a 30-min stabilized period was recorded to provide a temporally stable reference activity plateau (Fig. 1a, b). Subsequently, the networks generated relatively stable plateaus of spike activity after the application of each dose. Although different networks may have widely varying reference activities, the normalization process worked very well for each network and allowed for the plotting of the average concentrationresponse curves with minimal deviations (Fig. 1c).

L-Carnitine administered between 0.05 and $1.0 \mathrm{mM}$ induced a gradual, but discernible decrease in spike production (Fig. 1a). The spike activity was lost at a concentration of $2.0 \mathrm{mM}$. The active unit count, representing the number of discriminated units with more than ten threshold crossings/min, showed a delayed decrease with pronounced changes after the application of $0.6 \mathrm{mM}$ of L-carnitine. Following two complete washes, the electrophysiological activity was fully restored to the reference level, i.e., with a $100 \%$ recovery of active units. The concentration response data from networks exposed to L-carnitine are depicted in Fig. 1c. The mean $\mathrm{EC}_{50}$ was $0.22( \pm 0.01) \mathrm{mM}(n=6)$. D-Methionine also showed a stepwise decrease in spike activity (Fig. 1b). At a concentration of $10 \mathrm{mM}$, spike activity was reduced to $<10 \%$ of the reference level, while more than half of the selected neuronal units were still active, but fully recovered with two medium changes (W; Fig. 1b). The $\mathrm{EC}_{50}$ value for $\mathrm{D}$-methionine was calculated to be $1.06( \pm 0.06) \mathrm{mM}(n=6$; Fig. $1 \mathrm{c})$. 

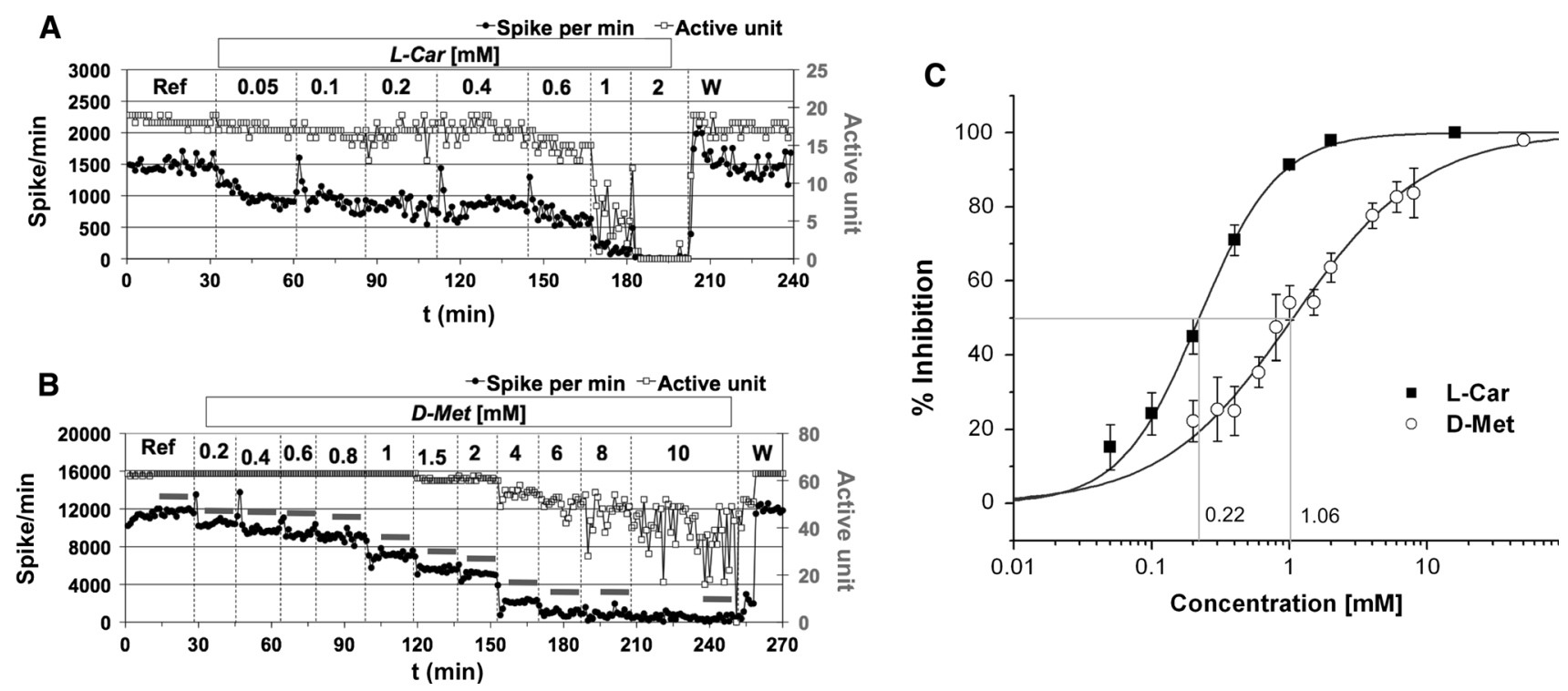

Fig. 1 Inhibition of neuronal activity by L-carnitine (L-Car; a) and D-methionine (D-Met, b) plotted as total network activity/min. Active units (right ordinate), defined by ten or more threshold crossings/min, are also plotted (open squares). In both cases, spike activity decreased before the loss of units. The stepwise activity decreases as a function of drug concentration are demonstrated for D-Met (b) where constant level plateau values were reached within $4.0 \mathrm{~min}$. L-Carnitine has a longer transitional period to quasi-stable plateaus. Bars above the

Network responses to L-carnitine and D-methionine in the presence of the $\mathrm{GABA}_{\mathrm{A}}$ antagonist bicuculline

Bicuculline is a competitive antagonist of the ionotropic $\mathrm{GABA}_{\mathrm{A}}$ receptor. In networks pretreated (30-60 min) with $40-\mu \mathrm{M}$ bicuculline, higher concentrations of $\mathrm{L}$-carnitine and D-methionine were needed to reach a $50 \%$ inhibition level (Fig. 2a, b). L-Carnitine at a concentration of $2.0 \mathrm{mM}$, which completely inhibited spike activity and active units in networks without bicuculline treatment, caused a $50 \%$ decrease in spike activity and a $20 \%$ decrease in active unit counts (Fig. 2a). However, a $90 \%$ inhibition level occurred at a concentration of $50 \mathrm{mM}$ (data not shown). D-Methionine titration was affected also by the presence of $40-\mu \mathrm{M}$ bicuculline. D-Methionine of $1.0 \mathrm{mM}$ concentration, which caused a $50 \%$ spike reduction in networks that were not exposed to bicuculline, had no effect on spike or unit reduction (Fig. 2b). The $\mathrm{EC}_{50}$ values for D-methionine ranged from 8 to $12 \mathrm{mM}$ in the presence of $40-\mu \mathrm{M}$ bicuculline. The active unit count remained unaffected by the increased D-methionine concentration (up to $50 \mathrm{mM}$ ). Despite the high concentrations used, two medium changes to fresh serum-free DMEM completely restored the spontaneous electrophysiological activity of both drugs to the native, non-bicuculline level (Fig. 2a, b). This recovery was characterized by the percent change relative to the native spike activity, with full recovery to $100 \%$ (Fig. 2c). To test spike plot in $\mathbf{b}$ indicate time periods from which plateau values were determined. $W$ Wash with fresh medium that returned the activity to reference levels in both cases. c Concentration-response of L-Car (filled squares $n=6$ ) or D-Met (open circles $n=6$ ) normalized as \% inhibition of spike activity and fitted by a sigmoidal algorithm. The $\mathrm{EC}_{50}$ for L-Car and D-Met are $0.22( \pm 0.01)$, and $1.06( \pm 0.06) \mathrm{mM}$, respectively

whether complete recovery of network spike activity from high concentrations of L-carnitine or D-methionine was achievable without bicuculline pre-treatment, single applications of 24 and $50 \mathrm{mM}$ (for $30 \mathrm{~min}$ ) were used. The inhibitory actions of both drugs were highly reversible with percent recovery that was not statistically different from $100 \%$ (Fig. 2c). No visible morphological changes of the cultures were observed under these high concentrations of L-carnitine or D-methionine (Fig. 2d, e).

Network burst patterns under L-carnitine and Dmethionine

The shift of potency for L-carnitine and D-methionine by bicuculline suggests agonist-antagonist interactions at the $\mathrm{GABA}_{\mathrm{A}}$ receptor. Total and rapid reversibility of the neuronal spike activity following complete medium replacement (wash) also showed that L-carnitine and D-methionine may be exerting their effects via membrane receptor activation. To further elucidate the agonist action of the $\mathrm{GABA}_{\mathrm{A}}$ receptor(s), we compared the change in network burst patterns under L-carnitine and D-methionine to that under muscimol, a potent $\mathrm{GABA}_{\mathrm{A}}$ agonist. The raster plot displays of sample spontaneous spike-train sequence of typical networks under serumfree DMEM are shown in Fig. 3a, b. Each spike cluster, with coordinated firing of multiple units, forms the burst activity in the networks. To quantify the pattern, we used two 

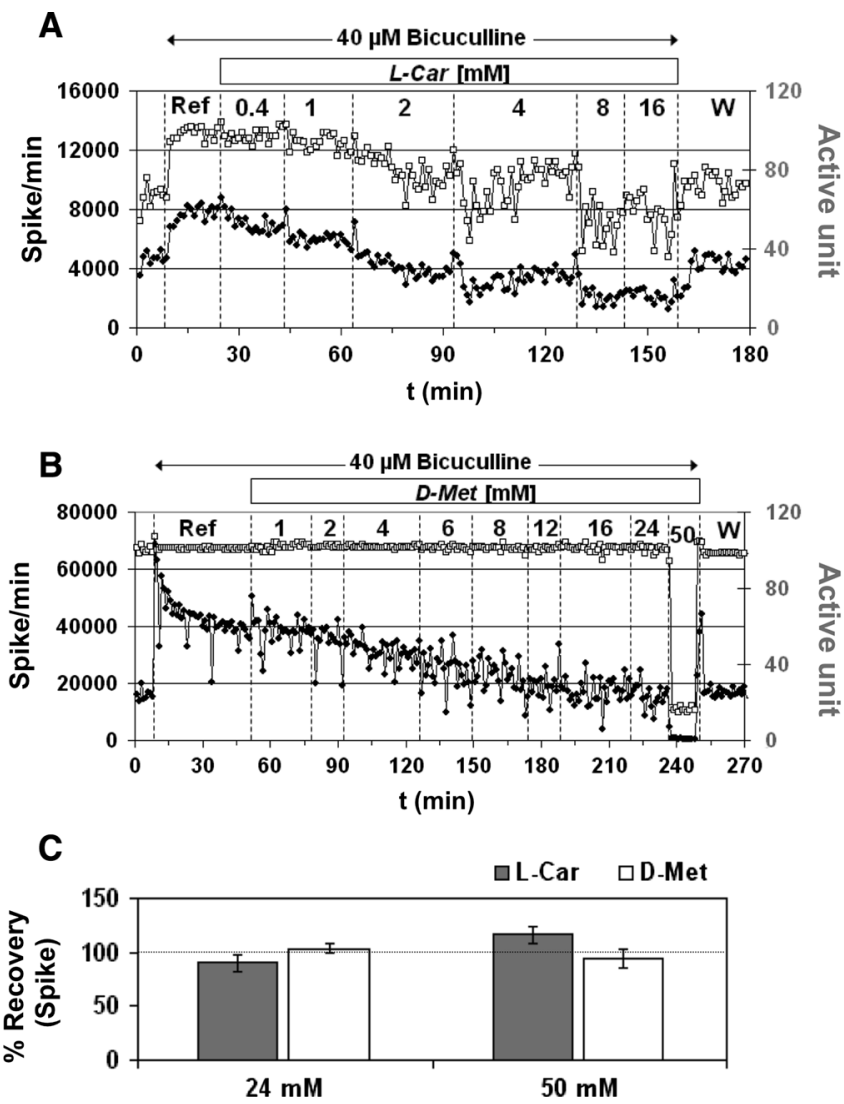

Fig. 2 Inhibition of neuronal activity by L-carnitine (L-Car) and D-methionine (D-Met) in the presence of bicuculline. a-b Stepwise decrease in per-min spike activity (left $y$-axis) and active unit count (right $y$-axis) with L-Car and D-Met titration in the presence of $40-\mu \mathrm{M}$ bicuculline. Note the lack of inhibition by the networks in the presence of bicuculline. $\mathbf{c}$ Percent recovery of spike activity for L-Car

parameters-burst period and burst duration. Burst period defines the interval from the beginning of a burst to the beginning of the next burst; burst duration marks the time span of spike occurrence within a burst. For both L-carnitine and D-methionine, the burst period increased as activity decreased stepwise with an increase in the concentrations of the drugs, while burst duration decreased as an indication of diminished burst strength (Fig. 3c, d).

From an analysis of previously published data (Rijal and Gross 2008), muscimol at its $\mathrm{EC}_{50}$ of $0.14 \mu \mathrm{M}$ increased the burst period by $2.3 \pm 0.6$-fold and at its $\mathrm{EC}_{90}(0.5 \mu \mathrm{M})$ by $40.3 \pm 14.2$-fold (Fig. 3e). When the networks were pretreated with $40-\mu \mathrm{M}$ bicuculline, the burst period increases were not observed. Relative fold changes of burst period under L-carnitine and D-methionine at both the $\mathrm{EC}_{50}$ and the $\mathrm{EC}_{90}$ are comparable to the data generated by muscimol $\left(F_{2,22}=0.01, \quad P=0.99\right.$ and $F_{2,14}=3.5, \quad P=0.064$, respectively). However, the change in the burst duration pattern exhibited pronounced differences between L-carnitine, D-methionine, and muscimol (Fig. 3f). At their
D

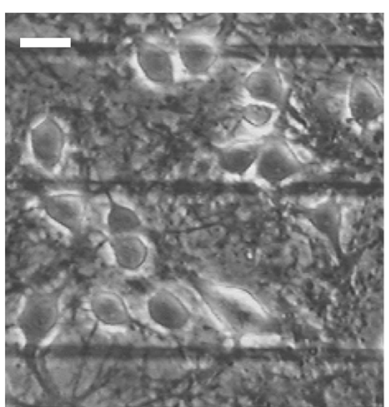

Ref

E

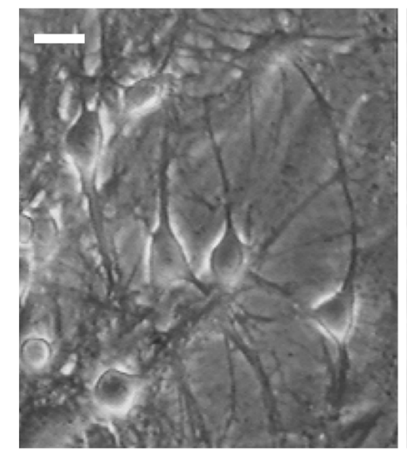

Ref

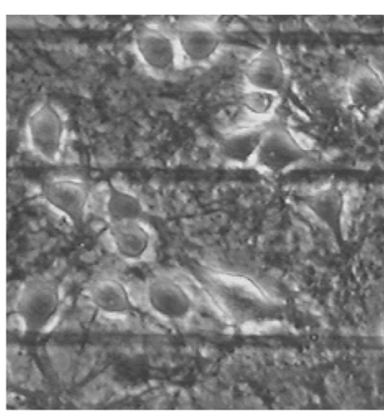

$50 \mathrm{mM} \mathrm{L-Car}$

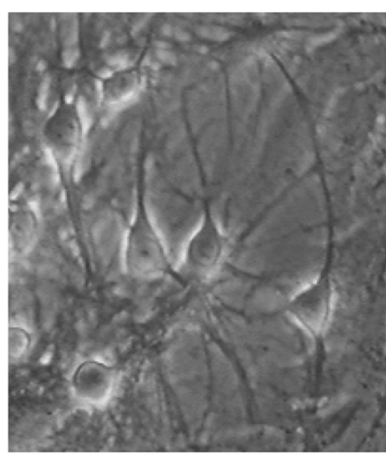

$50 \mathrm{mM}$ D-Met
(24 mM, $n=5 ; 50 \mathrm{mM}, n=5)$ and D-Met $(24 \mathrm{mM}, n=6 ; 50 \mathrm{mM}$, $n=3$ ) following two washes after a 30-min application. d-e Phasecontrast images of neuronal morphology under native condition (Ref), $50 \mathrm{mM} \mathrm{L}$-Car or D-Met for $30 \mathrm{~min}$. Bar $20 \mu \mathrm{m}$ for all panels. Horizontal lines in $\mathbf{d}$ represent insulated indium-tin oxide conductors outside the recording electrode matrix

respective $\mathrm{EC}_{50}$ values, the burst duration changes by L-carnitine and D-methionine were comparable to that by muscimol $\left(F_{2,20}=0.41, P=0.67\right)$. At the $\mathrm{EC}_{90} \mathrm{~s}$, the effect of muscimol was different (Tukey's post hoc, $P<0.05$ ) for both L-carnitine and D-methionine $\left(F_{2,15}=5.46\right.$, $P=0.012)$ as no reduction in burst duration was observed while $\mathrm{L}$-carnitine and D-methionine induced $-26.7 \%( \pm 5.2)$ and $-29.6( \pm 6.0)$ changes. Nevertheless, no burst changes were observed in the presence of $40-\mu \mathrm{M}$ bicuculline. Thisas well as the resemblance of network activity changes to muscimol -indicated a possible involvement of the $\mathrm{GABA}_{\mathrm{A}}$ receptor in the modulation of neuronal network burst properties by L-carnitine and D-methionine.

\section{Quantification of antagonism at the $\mathrm{GABA}_{\mathrm{A}}$ receptor}

To determine whether the shifts in potency are concentration dependent, various concentrations of bicuculline ranging from 0.5 to $40 \mu \mathrm{M}$ were used with titrations of L-carnitine and D-methionine (Fig. $4 \mathrm{a}, \mathrm{b}$ ). The $\mathrm{EC}_{50}$ value 

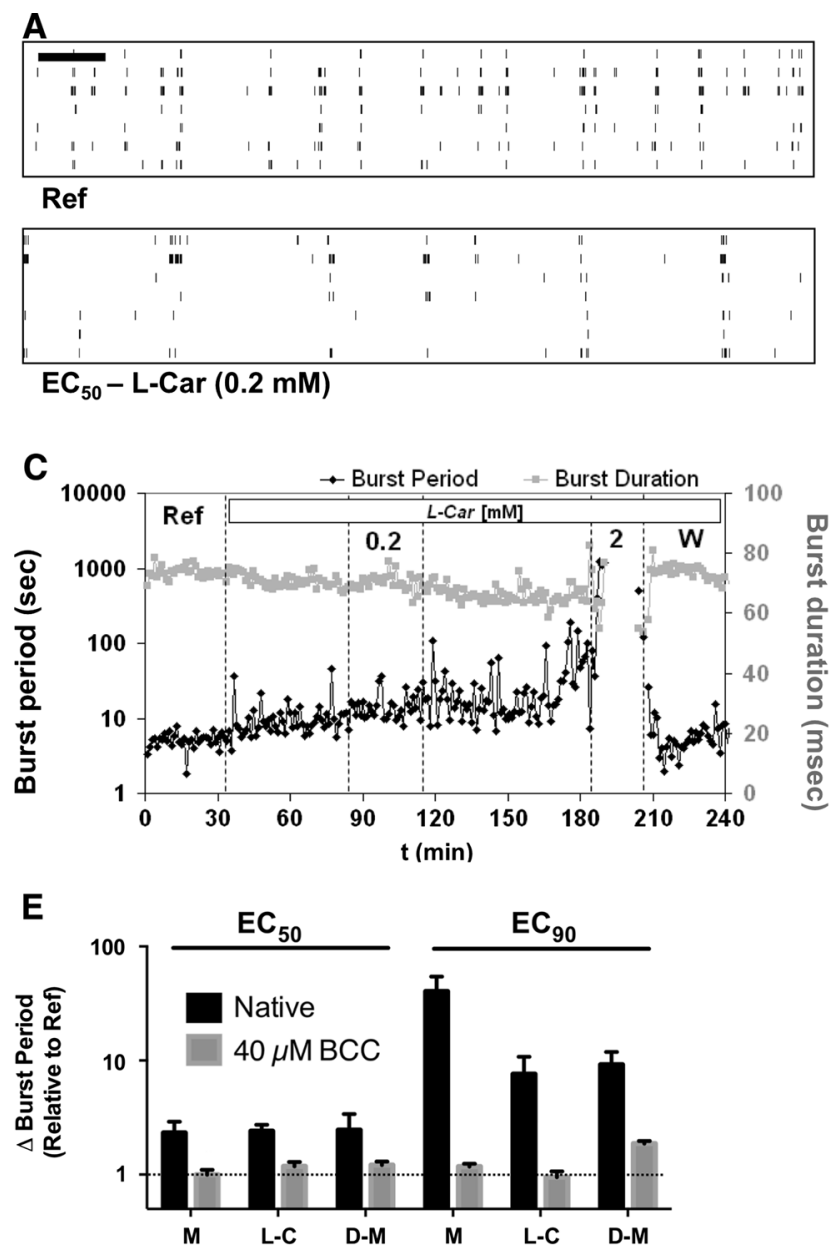

Fig. 3 Network response pattern under L-carnitine (L-Car) and D-methionine (D-Met). a-b Raster plot displays $35 \mathrm{~s}$ of spontaneous spike and burst activities from samples of seven units under native condition, and the $\mathrm{EC}_{50}$ condition for L-Car (a) and D-Met (b), bar $3 \mathrm{~s}$. c-d Burst parameters quantified as average burst period (left $y$-axis) and average burst duration (right $y$-axis) as a function of time.

of L-carnitine under $1.0 \mu \mathrm{M}$ bicuculline was shifted from 0.22 to $0.42 \mu \mathrm{M}$, although the Hill slope $\left(n_{\mathrm{H}}\right)$ did not exhibit a significant change (Fig. 4a; Table 1). Bicuculline at a concentration of $5.0 \mu \mathrm{M}$ caused an eightfold increase in the $\mathrm{EC}_{50}$, as well as a substantial reduction of $n_{\mathrm{H}}$. For concentrations of bicuculline of 10 and $40 \mu \mathrm{M}$, there was no significant change in the slope function and only a small increase of the $\mathrm{EC}_{50}$ was observed. In contrast to the effect on L-carnitine, bicuculline shifted the D-methionine concentration-response curves in a more stepwise manner, with less variability in $n_{\mathrm{H}}$, indicating a better-defined agonist-antagonist relationship (Fig. 4b). In the presence of bicuculline at $40 \mu \mathrm{M}$, the $\mathrm{EC}_{50}$ values of L-carnitine and D-methionine both exhibited a greater than tenfold increase, without affecting efficacy. Lineweaver-Burk plots were used to define the behavior of the antagonist (Fig. 4c-d; Emaduddin and Takeuchi 1996). For both

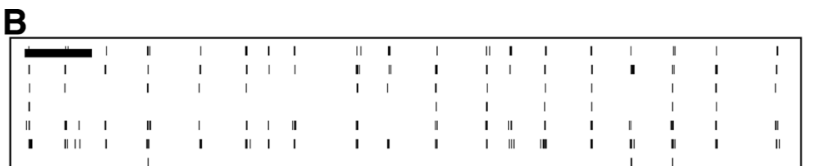

\section{Ref}
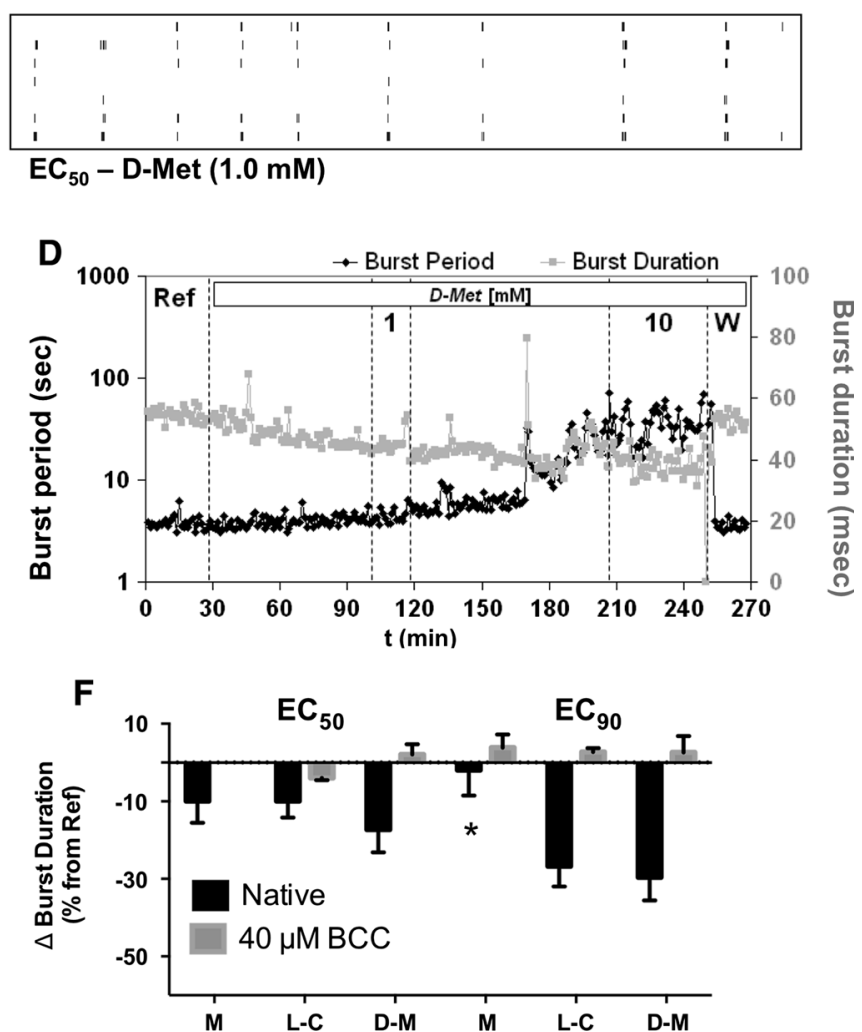

Timeline of L-Car or D-Met applications corresponds to Fig. 1a, b. Change in burst period (e) and burst duration (f) under muscimol (M), L-Car (L-C), D-Met (D-M) at $\mathrm{EC}_{50}(0.14 \mu \mathrm{M}, 0.2 \mathrm{mM}, 1.0 \mathrm{mM}$, respectively) and $\mathrm{EC}_{90}(0.5 \mu \mathrm{M}, 4.0 \mathrm{mM}, 12 \mathrm{mM}$, respectively). Significance indicates difference of drug response among M, L-C, and D-M; $* P<0.05$

L-carnitine and D-methionine responses, bicuculline predominately increased the linear slopes with only minor changes of the $y$-intercept values (Table 1) - a characteristic that supports competitive antagonism.

The $\mathrm{EC}_{50}$ shift as a function of antagonist concentration can therefore be plotted to determine dissociation constants by modified Gaddum equations (Gaddum 1957; Lew and Angus 1995). The $\mathrm{pEC}_{50}$ values of both compounds were extrapolated and plotted against the molar concentration of bicuculline (Fig. 5a) and the nonlinear regression parameters listed (Table 2). For competitive interaction between agonist and antagonist, the slope is defined to be 1.0. The dissociation constant (pA2) of bicuculline calculated by both the agonists L-carnitine and D-methionine were comparable -5.7 and 5.2, respectively (Table 2 ). The data of D-methionine has a lower goodness-of-fit value $\left(R^{2}\right)$ than that of L-carnitine (0.67 compared to 0.90 , respectively), 
Fig. 4 Concentration-response curves of L-carnitine (L-Car; $n=3-6$ ) (a) and D-methionine (D-Met, $n=5-6$ ) (b) in the presence of increasing concentrations of bicuculline (BCC; $\mu \mathrm{M})$. Summary of $\mathrm{EC}_{50}$ and Hill slope values are listed in Table 1. Same data plotted as reciprocal of L-Car (c) or D-Met (d) concentration $(\mathrm{mM})^{-1}$ vs. reciprocal of activity (\% Inhibition) $^{-1}$ using the Lineweaver-Burk method
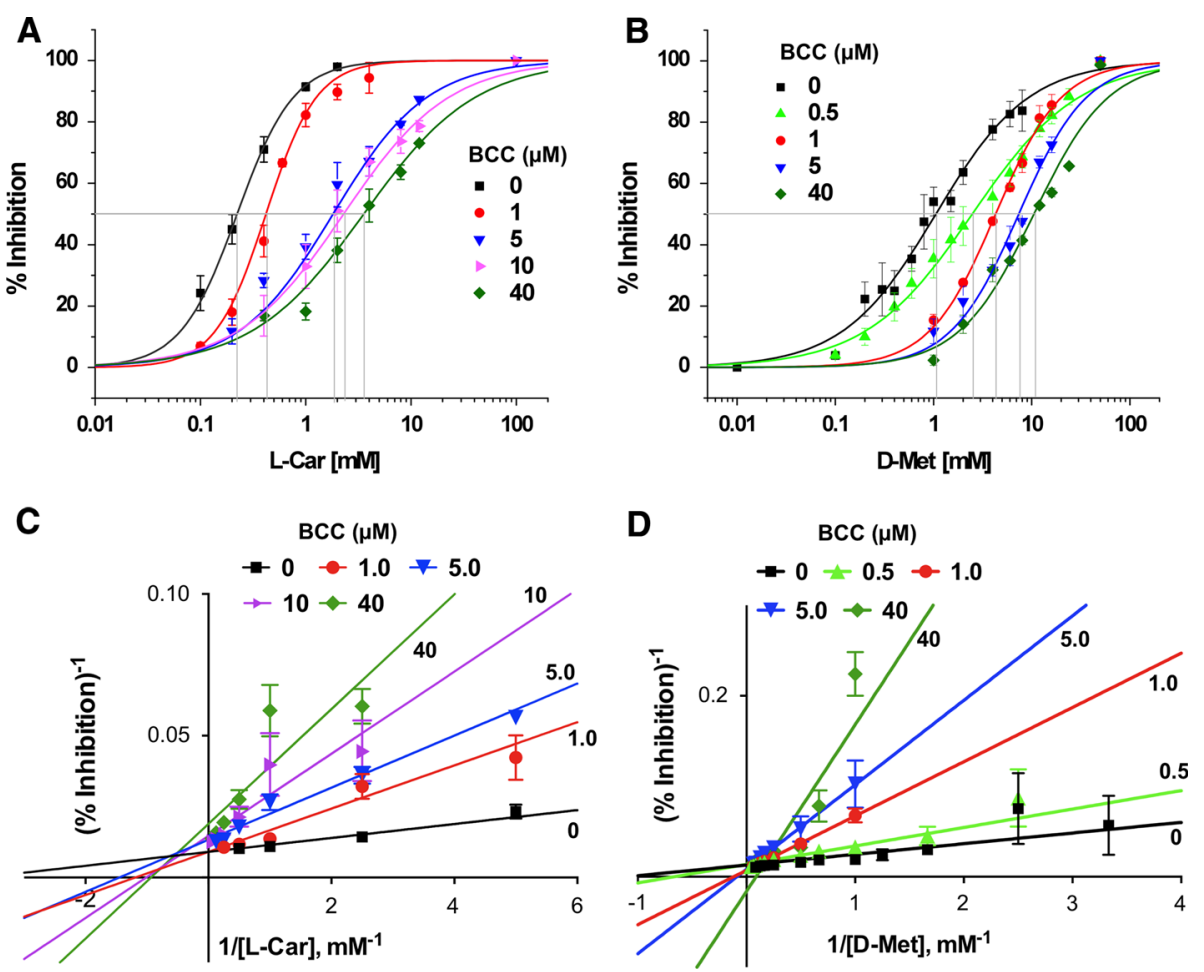

Table 1 Summary of $\mathrm{EC}_{50}$, Hill value $\left(n_{\mathrm{H}}\right)$, linear slope and $y$-intercept values (LineweaverBurk plots) for L-carnitine (L-Car) or D-methionine (D-Met) in the presence of increasing concentrations of bicuculline

\begin{tabular}{|c|c|c|c|c|c|c|}
\hline $\mathrm{BCC}(\mu \mathrm{M})$ & 0 & 0.5 & 1.0 & 5 & 10 & 40 \\
\hline \multicolumn{7}{|l|}{ L-Car } \\
\hline $\mathrm{EC}_{50}(\mathrm{mM})$ & $0.22 \pm 0.01$ & & $0.42 \pm 0.01$ & $1.82 \pm 0.32$ & $2.30 \pm 0.34$ & $3.57 \pm 0.26$ \\
\hline$n_{\mathrm{H}}$ & $1.62(n=6)$ & & $1.81(n=6)$ & $0.97(n=5)$ & $0.87(n=5)$ & $0.82(n=5)$ \\
\hline Linear slope & $0.03 \pm 0.01$ & & $0.01 \pm 0.001$ & $0.009 \pm 0.001$ & $0.015 \pm 0.004$ & $0.021 \pm 0.004$ \\
\hline$Y$-int & $0.01 \pm 0.00$ & & $0.01 \pm 0.003$ & $0.013 \pm 0.001$ & $0.015 \pm 0.004$ & $0.018 \pm 0.004$ \\
\hline \multicolumn{7}{|l|}{ D-Met } \\
\hline $\mathrm{EC}_{50}(\mathrm{mM})$ & $1.06 \pm 0.06$ & $2.48 \pm 0.15$ & $4.29 \pm 0.15$ & $7.39 \pm 0.52$ & & $10.52 \pm 0.97$ \\
\hline$n_{\mathrm{H}}$ & $0.92(n=6)$ & $0.80(n=6)$ & $1.26(n=4)$ & $1.25(n=3)$ & & $1.15(n=4)$ \\
\hline Linear slope & $0.01 \pm 0.00$ & $0.02 \pm 0.00$ & $0.06 \pm 0.00$ & $0.09 \pm 0.02$ & & $0.18 \pm 0.03$ \\
\hline$Y$-int & $0.01 \pm 0.01$ & $0.01 \pm 0.00$ & $0.01 \pm 0.00$ & $0.01 \pm 0.01$ & & $0.02 \pm 0.02$ \\
\hline
\end{tabular}

which may suggest that the pA2 value of 5.2 calculated from D-methionine was prone to a larger margin of error (Table 2). Figure 5b shows the linear relations using the $\log -\log$ Clark plot of the modified Gaddum equation. These pA2 values were lower than that calculated by muscimol (6.2), but approaching the range in the results of the literature (5.7-6.2; Rijal and Gross 2008). Our results may suggest competitive antagonism for both drugs when bicuculline is present at the putative $\mathrm{GABA}_{\mathrm{A}}$ receptor.

\section{Discussion}

L-Carnitine and D-methionine are both antioxidant compounds, but with different therapeutic implications. L-Carnitine is endogenously produced, derived from dietary sources, and is present in all tissue as a mitochondrial lipid transport carrier and energy precursor and, thus, plays important roles in diverse systemic disorders such as liver disease, kidney disease, endocrine disorders, and neurodegenerative diseases (Vaz and Wanders 2002; Silva-Adaya et al. 2008; Flanagan et al. 2010). D-Methionine is not endogenously present, and it has only been linked to ototoxic protection in recent years (Campbell et al. 2007; Dinh and Water 2009). Nonetheless, studies of the actions of both compounds in combating degenerative disorders have relied on the common mechanism of antioxidant activity as the basis for their potential use as neuro- and otoprotective agents (Gülçin 2006; Samson et al. 2008).

Our previous studies were among the first to adapt functional studies of neuronal networks for testing the 
A

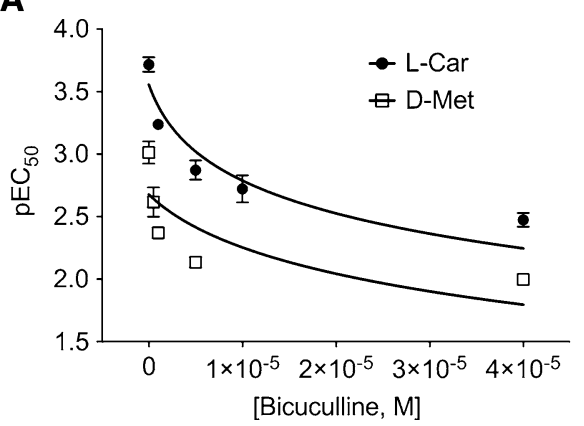

Fig. 5 Extrapolated $\mathrm{pEC}_{50}$ values graphed as function of bicuculline concentrations. a Logarithmic regression curves are fitted with equation $\mathrm{pEC}_{50}=-\log \left([B]+10^{-\mathrm{pA} 2}\right)-C$ for computation of pA2 values. Curve fitting parameters are listed in Table 2. b Clark

Table 2 Summary of nonlinear regression fits (re: L-carnitine and Dmethionine) for the equation $\mathrm{pEC}_{50}=-\log \left([B]+10^{-\mathrm{pA} 2}\right)-C$, where $[B]$ is the bicuculline concentration and $\mathrm{pA} 2$ is the dissociation constant

\begin{tabular}{lll}
\hline & L-Carnitine & D-Methionine \\
\hline pA2 & $5.69 \pm 0.12$ & $5.22 \pm 0.22$ \\
$C$ & $2.13 \pm 0.07$ & $0.22 \pm 0.18$ \\
$R^{2}$ & 0.898 & 0.671 \\
\hline
\end{tabular}

$C$ is a fitting constant (see Lew and Angus 1995)

protective properties of L-carnitine and D-methionine (Wu et al. 2011; Gopal et al. 2012). We observed that both compounds elicited consistent inhibitory responses in neuronal networks. However, neuronal modulatory properties such as that which is seen in the presence of GABAergic agents were not emphasized previously. We have now shown that the effect of L-carnitine and D-methionine resemble agonists of the $\mathrm{GABA}_{\mathrm{A}}$ receptor. The hypothesis is supported by the clear shift of their concentrationresponse curves in the presence of bicuculline, a $\mathrm{GABA}_{\mathrm{A}}$ receptor antagonist. The Lineweaver-Burk plot analysis showed that the shifts in potency of L-carnitine and D-methionine in the presence of bicuculline might be due to a mechanism or mechanisms of competitive antagonism. Other types of antagonistic behaviors may also be possible, as the Lineweaver-Burk plots exhibited minor shifts in the $y$-intercepts and the Hill slopes of the concentrationresponse curves were non-uniform. Using nonlinear regression, the calculated dissociation constant of bicuculline was well within the established values present in the literature (see Rijal and Gross 2008), which further suggested the roles of L-carnitine and D-methionine as $\mathrm{GABA}_{\mathrm{A}}$ activators. The patterns of spike inhibition (for both compounds) also resembled that elicited by the $\mathrm{GABA}_{\mathrm{A}}$ agonist muscimol. Furthermore, high $(<50 \mathrm{mM})$ concentrations of the compounds did not cause morphological damage to the
B

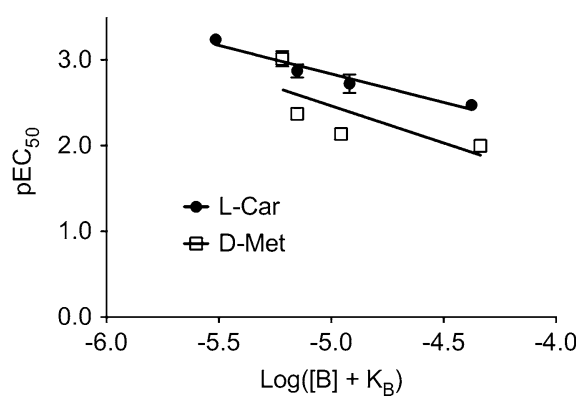

plot display and linear relations of the $\mathrm{pEC}_{50}$ values graphed as function of $\log$ bicuculline concentration $[B]$, adjusted with the calculated dissociation constants $\left(K_{\mathrm{B}}=-\log (\mathrm{pA} 2)\right)$

networks and the effects were completely reversible electrophysiologically. The resultant rapid responses are highly stereotypic of plasma membrane receptor function. Nevertheless, only ligand-binding assays may offer direct evidence of physical interaction between the two antioxidant compounds and bicuculline at the $\mathrm{GABA}_{\mathrm{A}}$ site (Smith and Simpson 2003).

A few studies have already linked L-carnitine to neuronal GABAergic modulation. An alternative form, acetyl-Lcarnitine, the active metabolite formed by free L-carnitine and acetyl-CoA, elevates GABA concentration in mouse substantia nigra in vivo (Fariello et al. 1988). Two separate studies concluded that acetyl-L-carnitine is involved in the production of releasable glutamate (a GABA precursor), as well as GABA quantification through bioradiography imaging (Tanaka et al. 2003) and NMR spectroscopy (Scafidi et al. 2010). Our results do not rule out the role of L-carnitine in neurotransmitter synthesis, yet the reported increase in GABA concentrations cannot fully account for the large, persistent inhibitory patterns that we observed in this study. Increases in synaptic GABA concentration reported in the aforementioned studies occur on the time scale between hours to days; whereas we observed an almost immediate response to L-carnitine administration (Fig. 1a).

Discernible electrophysiological responses of neurons to L-carnitine, thus, may be linked to plasma membrane receptor modulations. Lombardo et al. (2004) observed an increase in membrane after-hyperpolarization upon administration of $50 \mu \mathrm{M}$ to $2.0 \mathrm{mM}$ acetyl-L-carnitine, recorded from segmental ganglion neurons of adult leeches. Computational modeling of the acetyl-L-carnitineinduced effects resulted in a systemic decrement in action potential production (Lombardo et al. 2004). This conclusion, albeit implying a possible potassium effect (afterhyperpolarization) of L-carnitine, can also support our observations from direct neuronal network recordings 
using the microelectrode array system. This platform of network electrophysiology, moreover, enabled us to observe patterns of neuronal activity that are not accessible through, e.g., single-channel techniques (Rijal and Gross 2008; Johnstone et al. 2010).

It is essential to note that bicuculline, other than its specific action of competitive $\mathrm{GABA}_{\mathrm{A}}$ antagonism, also antagonizes the nicotinic acetylcholine receptor at higher concentrations (about tenfold higher than its $\mathrm{IC}_{50}$ of GABA $_{\mathrm{A}}$ binding; Demuro et al. 2001). L-Carnitine plays an intricate role in acetylcholine metabolism as well as stimulates cholinergic neurotransmission (Nalecz et al. 2004; Di Cesare Mannelli et al. 2009). It is unlikely that nicotinic acetylcholine receptors were primarily responsible for the bicuculline-mediated curve shift, since nicotinic acetylcholine receptor modulation produce minimal activity change. We recognize also that there may be undetected cholinergic contribution to L-carnitine-mediated activity inhibition.

D-Methionine has a more obscure mechanistic profile. The cytoprotective properties were identified by the action of the thiol group for free radical scavenging (Vogt 1995). Major findings on the effect of D-methionine were obtained in vivo (Campbell et al. 2003) and specifically against noise or chemical (especially cisplatin)-induced oxidative insult on inner ear cochlear tissues. Campbell et al. (2003) alluded to the fact that the protective properties of D-methionine may in part originate from its ability to increase endogenous antioxidant concentrations, but others also suggested the direct chelation of D-methionine to platinum toxins (Ekborn et al. 2002). As the effect of Dmethionine or its protective action against platinum toxicity on electrophysiologically active neurons was uncharted, our studies suggesting $\mathrm{GABA}_{\mathrm{A}}$ involvement provides a heretofore novel perspective.

Another possible mechanism for D-methionine-induced activity suppression may be a secondary effect of glutathione. The thiol-containing reducing agent, directly acting as a scavenger for ROS, also plays a role in redox modulation of the $\mathrm{GABA}_{\mathrm{A}}$ receptor and activation of $\mathrm{Cl}^{-}$currents (Amato et al. 1999; Wilkins and Smart 2002). However, glutathione and other reducing antioxidants failed to induce a network inhibition (preliminary data, not shown). Glutathione is also implicated in the metabolism of glutamate (Koga et al. 2011), but as in the case for L-carnitine, metabolic contributions of neurochemicals do not produce very rapid concentration-dependent network responses, as can be seen with direct receptor activation. However, it must be noted that in our neuronal/glial coculture system - a benefit as it more closely resembles the in vivo state-astrocytic $\mathrm{GABA}_{\mathrm{A}}$ receptors (Liu et al. 1996) may also be targets of $\mathrm{L}$-carnitine or $\mathrm{D}$-methionine, but possible astrocytic $\mathrm{GABA}_{\mathrm{A}}$ receptor contributions to these compounds' neuromodulatory effects require further studies.

The promises and benefits of L-carnitine and D-methionine are already extending to clinical treatment of various neurological disorders. Thus, the mechanism of GABAergic modulation could perhaps serve to better explicate the role of $\mathrm{L}$-carnitine and D-methionine in particular diseases. $\mathrm{GABA}_{\mathrm{A}}$ agonist actions, for instance, may be a direct cause of L-carnitine's analgesic effect in neuropathy (Chiechio et al. 2007). Anesthetic-induced damage presented as potentiation of the $\mathrm{GABA}_{\mathrm{A}}$ receptor, perhaps, was rescued by L-carnitine due to its competition for receptor binding with isoflurane (Zou et al. 2008). The concept of excitotoxicity, the disturbance of membrane ionic balance that leads to degeneration-such as that induced by excess glutamate in Huntington Disease symptoms or methamphetamine-activated dopamine surge (Virmani et al. 2003; Vamos et al. 2010) - may be more relevant in the discussion of the protective action of L-carnitine within the framework of the inhibitory neurotransmitter system. Likewise, we suspect that perhaps the protective mechanism of D-methionine against platinum toxicity observed in our previous study (Gopal et al. 2012) may partly be a counteracting measure against excitotoxicity by GABAergic inhibition.

In conclusion, we presented evidence that the antioxidants L-carnitine and D-methionine may modulate neuronal activity through reversible binding to the $\mathrm{GABA}_{\mathrm{A}}$ receptor(s), causing inhibition of spontaneous neuronal network activity. The agonist actions were competitively antagonized by bicuculline. Thus, our study presents a novel finding that not only contributes to the understanding of the mechanisms of L-carnitine and D-methionine, but also reiterates the importance of the GABAergic system in neurotransmission and neuroprotection. Moreover, as the search for and the development of neuroprotective compounds become an important aspect of neurological and otological research, we demonstrated that the in vitro neuronal network MEA platform serves as an effective network model for screening and investigation of new drug actions. We suspect that direct observations of changes in a dynamic, spontaneously active neuronal network, such as that demonstrated in this study, will be increasingly accepted as a unique and informative interrogation of the simultaneous manifestation of several pharmaceutical mechanisms.

Acknowledgments The authors thank Ahmet Ors for expert fabrication of microelectrode arrays, and Nga Nguyen for excellent and unrelenting cell culture support. S. Rijal provided raw data for the muscimol experiments. The research was supported in part by the Charles Bowen Endowment to the Center for Network Neuroscience (GG), by internal UNT Startup funds (EJM), and by financial support provided by the Once Upon a Time Foundation (EJM). 
Conflict of interest The authors declare that they have no conflict of interest.

\section{References}

Amato A, Connolly CN, Moss SJ, Smart TG (1999) Modulation of neuronal and recombinant $\mathrm{GABA}_{\mathrm{A}}$ receptors by redox reagents. J Physiol 517:35-50

Blakley BW, Cohen JI, Doolittle ND, Muldoon LL, Campbell KC, Dickey DT, Neuwelt EA (2002) Strategies for prevention of toxicity caused by platinum-based chemotherapy: review and summary of the annual meeting of the blood-brain barrier disruption program, Gleneden Beach, Oregon, March 10, 2001. Laryngoscope 112:1997-2001

Campbell KC, Meech RP, Rybak LP, Hughes LF (2003) The effect of D-methionine on cochlear oxidative state with and without cisplatin administration: mechanisms of otoprotection. J Am Acad Audiol 14:144-156

Campbell KC, Meech RP, Klemens JJ, Gerberi MT, Dyrstad SS, Larsen DL, Mitchell DL, El-Azizi M, Verhulst SJ, Hughes LF (2007) Prevention of noise- and drug-induced hearing loss with D-methionine. Hear Res 226:92-103

Chiechio S, Copani A, Gereau RW, Nicoletti F (2007) Acetyl-Lcarnitine in neuropathic pain: experimental data. CNS Drugs 21(Suppl 1):31-38

Demuro A, Palma E, Eusebi F, Miledi R (2001) Inhibition of nicotinic acetylcholine receptors by bicuculline. Neuropharmacology 41:854-861

Di Cesare Mannelli L, Ghelardini C, Calvani M, Nicolai R, Mosconi L, Toscano A, Pacini A, Bartolini A (2009) Neuroprotective effects of acetyl-L-carnitine on neuropathic pain and apoptosis: a role for the nicotinic receptor. J Neurosci Res 87:200-207

Dinh CT, Van De Water TR (2009) Blocking pro-cell-death signal pathways to conserve hearing. Audiol Neurootol 14:383-392

Ekborn A, Laurell G, Johnström P, Wallin I, Eksborg S, Ehrsson H (2002) D-Methionine and cisplatin ototoxicity in the guinea pig: D-methionine influences cisplatin pharmacokinetics. Hear Res 165:53-61

Emaduddin M, Takeuchi H (1996) Lineweaver-Burk analysis for the blocking effects of mammalian dopamine receptor antagonists on dopamine-induced currents in Achatina giant neurones. Gen Pharmacol 27:1209-1213

Fariello RG, Ferraro TN, Golden GT, DeMattei M (1988) Systemic acetyl-L-carnitine elevates nigral levels of glutathione and GABA. Life Sci 43:289-292

Flanagan JL, Simmons PA, Vehige J, Willcox MD, Garrett Q (2010) Role of carnitine in disease. Nutr Metab (Lond) 7:30

Gaddum JH (1957) Theories of drug antagonism. Pharmacol Rev 9:211-218

Gopal KV, Gross GW (1996) Auditory cortical neurons in vitro: cell culture and multichannel extracellular recording. Acta Otolaryngol 116:690-696

Gopal KV, Miller BR, Gross GW (2007) Acute and sub-chronic functional neurotoxicity of methylphenidate on neural networks in vitro. J Neural Transm 114:1365-1375

Gopal KV, Wu C, Shrestha B, Campbell KC, Moore EJ, Gross GW (2012) D-Methionine protects against cisplatin-induced neurotoxicity in cortical networks. Neurotoxicol Teratol 34:495-504

Gross GW, Schwalm FU (1994) A closed chamber for long-term electrophysiological and microscopical monitoring of monolayer neuronal networks. J Neurosci Methods 52:73-85

Gross GW, Wen W, Lin J (1985) Transparent indium-tin oxide patterns for extracellular, multisite recording in neuronal cultures. J Neurosci Methods 15:243-252
Gülçin I (2006) Antioxidant and antiradical activities of L-carnitine. Life Sci 78:803-811

Henderson D, Bielefeld EC, Harris KC, Hu BH (2006) The role of oxidative stress in noise-induced hearing loss. Ear Hear 27:1-19

Johnstone AF, Gross GW, Weiss DG, Schroeder OH, Gramowski A, Shafer TJ (2010) Microelectrode arrays: a physiologically based neurotoxicity testing platform for the 21 st century. Neurotoxicology 31:331-350

Jones LL, McDonald DA, Borum PR (2010) Acylcarnitines: role in brain. Prog Lipid Res 49:61-75

Keefer EW, Gramowski A, Stenger DA, Pancrazio JJ, Gross GW (2001) Characterization of acute neurotoxic effects of trimethylolpropane phosphate via neuronal network biosensors. Biosens Bioelectron 16:513-525

Koga M, Serritella AV, Messmer MM, Hayashi-Takagi A, Hester LD, Snyder SH, Sawa A, Sedlak TW (2011) Glutathione is a physiologic reservoir of neuronal glutamate. Biochem Biophys Res Commun 409:596-602

Lew MJ, Angus JA (1995) Analysis of competitive agonist-antagonist interactions by nonlinear regression. Trends Pharmacol Sci 16:328-337

Liu QY, Schaffner AE, Li YX, Dunlap V, Barker JL (1996) Upregulation of GABAA current by astrocytes in cultured embryonic rat hippocampal neurons. J Neurosci 16:2912-2923

Lombardo P, Scuri R, Cataldo E, Calvani M, Nicolai R, Mosconi L, Brunelli M (2004) Acetyl-L-carnitine induces a sustained potentiation of the after hyperpolarization. Neuroscience 128:293-303

Lorito G, Hatzopoulos S, Laurell G, Campbell KC, Petruccelli J, Giordano P, Kochanek K, Sliwa L, Martini A, Skarzynski H (2011) Dose-dependent protection on cisplatin-induced ototoxicity-an electrophysiological study on the effect of three antioxidants in the Sprague-Dawley rat animal model. Med Sci Monit 17:179-186

Morefield SI, Keefer EW, Chapman KD, Gross GW (2000) Drug evaluations using neuronal networks cultured on microelectrode arrays. Biosens Bioelectron 15:383-396

Nalecz KA, Miecz D, Berezowski V, Cecchelli R (2004) Carnitine: transport and physiological functions in the brain. Mol Asp Med 25:551-567

Napolitano A, Manini P, d'Ischia M (2011) Oxidation chemistry of catecholamines and neuronal degeneration: an update. Curr Med Chem 18:1832-1845

Nisticò R, Mehdawy B, Piccirilli S, Mercuri N (2011) Paraquat- and rotenone-induced models of Parkinson's disease. Int J Immunopathol Pharmacol 24:313-322

Palacios HH, Yendluri BB, Parvathaneni K, Shadlinski VB, Obrenovich ME, Leszek J, Gokhman D, Gąsiorowski K, Bragin V, Aliev G (2011) Mitochondrion-specific antioxidants as drug treatments for Alzheimer disease. CNS Neurol Disord Drug Targets 10:149-162

Poirrier AL, Pincemail J, Van Den Ackerveken P, Lefebvre PP, Malgrange B (2010) Oxidative stress in the cochlea: an update. Curr Med Chem 17:3591-3604

Rijal SO, Gross GW (2008) Dissociation constants for GABA(A) receptor antagonists determined with neuronal networks on microelectrode arrays. J Neurosci Methods 173:183-192

Rybak LP (2005) Neurochemistry of the peripheral and central auditory system after ototoxic drug exposure: implications for tinnitus. Int Tinnitus J 11:23-30

Rybak LP, Whitworth CA, Mukherjea D, Ramkumar V (2007) Mechanisms of cisplatin-induced ototoxicity and prevention. Hear Res 226:157-167

Samson J, Wiktorek-Smagur A, Politanski P, Rajkowska E, Pawlaczyk-Luszczynska M, Dudarewicz A, Sha SH, Schacht J, 
Sliwinska-Kowalska M (2008) Noise-induced time-dependent changes in oxidative stress in the mouse cochlea and attenuation by D-methionine. Neuroscience 152:146-150

Scafidi S, Fiskum G, Lindauer SL, Bamford P, Shi D, Hopkins I, McKenna MC (2010) Metabolism of acetyl-L-carnitine for energy and neurotransmitter synthesis in the immature rat brain. J Neurochem 114:820-831

Sesti F, Liu S, Cai SQ (2010) Oxidation of potassium channels by ROS: a general mechanism of aging and neurodegeneration? Trends Cell Biol 20:45-51

Silva-Adaya D, Pérez-De La Cruz V, Herrera-Mundo MN, MendozaMacedo K, Villeda-Hernández J, Binienda Z, Ali SF, Santamaría A (2008) Excitotoxic damage, disrupted energy metabolism, and oxidative stress in the rat brain: antioxidant and neuroprotective effects of L-carnitine. J Neurochem 105:677-689

Smith AJ, Simpson PB (2003) Methodological approaches for the study of $\mathrm{GABA}(\mathrm{A})$ receptor pharmacology and functional responses. Anal Bioanal Chem 377:843-851

Tanaka M, Nakamura F, Mizokawa S, Matsumura A, Matsumura K, Watanabe Y (2003) Role of acetyl-L-carnitine in the brain: revealed by bioradiography. Biochem Biophys Res Commun 306:1064-1069

Vamos E, Voros K, Vecsei L, Klivenyi P (2010) Neuroprotective effects of L-carnitine in a transgenic animal model of Huntington's disease. Biomed Pharmacother 64:282-286
Vaz FM, Wanders RJ (2002) Carnitine biosynthesis in mammals. Biochem J 361:417-429

Virmani A, Gaetani F, Imam S, Binienda Z, Ali S (2003) Possible mechanism for the neuroprotective effects of L-carnitine on methamphetamine-evoked neurotoxicity. Ann N Y Acad Sci 1993:197-207

Vogt W (1995) Oxidation of methionyl residues in proteins: tools, targets, and reversal. Free Radic Biol Med 18:93-105

Waldbaum S, Patel M (2010) Mitochondria, oxidative stress, and temporal lobe epilepsy. Epilepsy Res 88:23-45

Wilkins ME, Smart TG (2002) Redox modulation of GABAA receptors obscured by $\mathrm{Zn}^{2+}$ complexation. Neuropharmacology 43:938-944

Wu C, Gopal K, Gross GW, Lukas TJ, Moore EJ (2011) An in vitro model for testing drugs to treat tinnitus. Eur $\mathrm{J}$ Pharmacol 667:188-194

Zou X, Sadovova N, Patterson TA, Divine RL, Hotchkiss CE, Ali SF, Hanig JP, Paule MG, Slikker W, Wang C (2008) The effects of L-carnitine on the combination of, inhalation anesthetic-induced developmental, neuronal apoptosis in the rat frontal cortex. Neuroscience 51:1053-1065 